\title{
Independent Coding of Reward Magnitude and Valence in the Human Brain
}

\author{
Nick Yeung and Alan G. Sanfey \\ Center for the Study of Brain, Mind, and Behavior, Department of Psychology, Princeton University, Princeton, New Jersey 08544
}

\begin{abstract}
Previous research has shown that two components of the event-related brain potential, the P300 and feedback negativity, are sensitive to information about rewards and penalties. The present study investigated the properties of these components in a simple gambling game that required participants to choose between cards that were unpredictably associated with monetary gains and losses of variable magnitude. The aim was to determine the sensitivity of each component to two critical features of reward stimuli: magnitude (small or large) and valence (win or loss). A double dissociation was observed, with the P300 sensitive to reward magnitude but insensitive to reward valence and the feedback negativity showing the opposite pattern, suggesting that these two fundamental features of rewarding stimuli are evaluated rapidly and separately in the human brain. Subsequent analyses provided additional evidence of functional dissociations between the feedback negativity and P300. First, the P300 (but not the feedback negativity) showed sensitivity to the reward value of alternative, nonselected stimuli. Second, individual differences in the amplitude of the feedback negativity correlated with individual differences in risk-taking behavior observed after monetary losses, whereas individual differences in P300 amplitude were related to behavioral adjustments observed in response to alternative, unchosen outcomes.
\end{abstract}

Key words: event-related potential; feedback; error-related negativity; medial frontal negativity; P300; decision making

\section{Introduction}

A central function of the cognitive system is to determine the affective or motivational significance of ongoing events, and it seems likely that mechanisms have evolved to provide rapid evaluations of the reward value of stimuli we encounter in our environment. Studies using electroencephalographic (EEG) recordings from human participants have identified event-related brain potential (ERP) correlates of this rapid evaluative function (Sutton et al., 1978; Johnston, 1979; Gehring and Willoughby, 2002; Holroyd and Coles, 2002). These experiments have typically involved participants performing simple gambling games in which they win or lose money on the basis of unpredictable outcomes.

Early studies reported that feedback stimuli indicating the outcomes of monetary gambles elicit a slow-wave component, labeled the P300 for its positive polarity and latency, that increases in amplitude with the amount of money won or lost (Sutton et al., 1978; Johnston, 1979). More recently, it has been shown that feedback stimuli also elicit a negative ERP component occurring 200-300 msec after feedback delivery. This feedback negativity is larger in amplitude after negative outcomes, such as incorrect responses or losses of money, than after positive outcomes (Miltner et al., 1997; Gehring and Willoughby, 2002; Holroyd and Coles, 2002), leading to the proposal that this com-

Received Aug. 18, 2003; revised May 3, 2004; accepted May 20, 2004.

This research was supported by National Institutes of Health Grant P50-MH62196 and by a grant from the Seaver Institute (A.G.S.). We thank Jonathan Cohen, Clay Holroyd, and Sander Nieuwenhuis for helpful discussions and comments on previous drafts of this manuscript.

Correspondence should be addressed to Nick Yeung, Department of Psychology, Green Hall, Princeton University, Princeton, NJ 08544. E-mail: nyeung@princeton.edu.

DOI:10.1523/JNEUROSCI.4537-03.2004

Copyright $\odot 2004$ Society for Neuroscience $\quad$ 0270-6474/04/246258-07\$15.00/0 ponent reflects the evaluation of events along a good-bad dimension.

Previous research has thus identified two ERP correlates of reward processing, but fundamental questions remain concerning the underlying cognitive and neural processes. First, it remains to be determined whether P300 amplitude varies with reward value simply because participants pay more attention to outcomes of larger monetary gambles, or whether P300 amplitude indexes meaningful changes in cognitive processing of reward information. Second, the nature of the evaluative process reflected in the feedback negativity remains unknown. One possibility is that the evaluation provides a scalar estimate of utility or value, in which case the feedback negativity should increase in amplitude with increases in monetary penalty. Alternatively, the evaluation might discretely classify events as being "good" or "bad" essentially independently of the magnitude of reward obtained or penalty incurred, in which case the feedback negativity should be insensitive to reward magnitude.

To address these issues, in the present research we recorded ERPs from human participants as they performed a simple monetary gambling game. The participants were required to select between two colored cards that were unpredictably associated with small and large monetary gains and losses. After their selection, participants were given feedback indicating the outcome of their choice and were then told what they would have won had they made the alternative choice. Using this design, we directly investigated how the valence and magnitude of experienced rewards affected the P300 and feedback negativity. In addition, the design allowed us to investigate whether these ERP components are sensitive only to immediate, experienced rewards, or whether they are also sensitive to the reward value of alternative, nonse- 


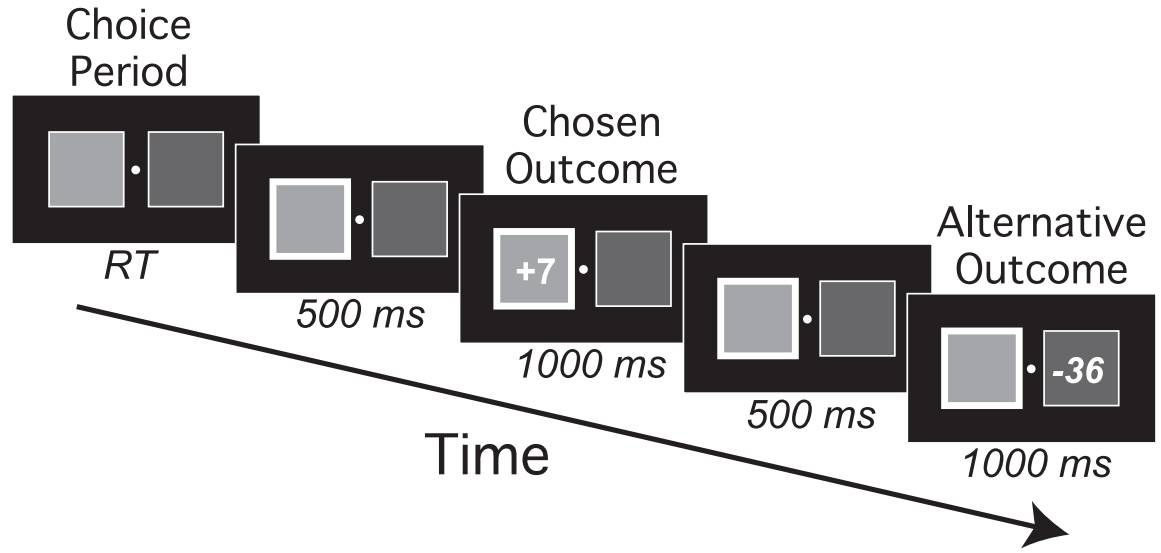

Figure 1. The sequence of events during a single trial of the gambling game. Participants were presented on each trial with a choice of two colored cards, one of which they selected with a left- or right-hand keypress. After $500 \mathrm{msec}$, they were shown the outcome associated with the selected card for $1000 \mathrm{msec}$. After an additional $500 \mathrm{msec}$, they were shown the alternative outcome (i.e., what they would have won had they made the alternative choice). RT, Response time.

lected choices. Finally, this design enabled us to investigate the relationship between ERP signatures of reward processing and participants' behavioral choices.

\section{Materials and Methods}

Participants. Sixteen right-handed participants (eight females and eight males, 18-22 years of age), all undergraduates at Princeton University, received course credit and a small monetary bonus for their participation. The monetary bonus depended on the outcomes of the gambling game, as described below. All participants gave written informed consent, and the study was approved by the local research ethics committee.

Procedure. The participants were seated comfortably $\sim 1 \mathrm{~m}$ in front of a computer screen in an electromagnetically shielded room. On each trial of the experiment (see Fig. 1 for an example), they were presented with two colored rectangles displayed on either side of a fixation point and were asked to imagine that these rectangles were cards dealt from a randomly shuffled deck containing cards of four colors. This pair of cards remained on the screen until the participant selected one of them by pressing a button with either their left or right index finger, corresponding to the location of the chosen card. After their response, the chosen card was highlighted with a thick white border for $500 \mathrm{msec}$, after which time a number (either positive or negative) appeared on the card for 1000 msec to indicate how much money they won or lost on the trial; this was the chosen outcome. After an additional interval of $500 \mathrm{msec}$, the participants were shown what they would have won or lost had they selected the other card. We called this the alternative outcome. Again, this information remained on the screen for $1000 \mathrm{msec}$ and was followed by an intertrial interval of $500 \mathrm{msec}$.

Four colors of cards were used: red, green, blue, and purple. Two of the colors were always associated with large outcomes, which were wins or losses of $32-40 \$$ (these colors were referred to as large decks), and two were always associated with small outcomes, which were wins or losses of $6-11 \$$ (these colors were referred to as small decks). The assignment of colors to reward magnitudes was varied across participants. The colors presented on any given trial were randomized with the constraint that each possible pairing occurred with equal frequency. Pairings of identical colored cards were allowed. Thus, on half of all trials, participants chose between one large card and one small card. The other half of trials involved selecting between two small cards or between two large cards.

The outcome of each trial was determined pseudorandomly with the constraint that each participant experienced equal numbers of small wins and small losses and equal numbers of large wins and large losses. The ratio of small to large outcomes depended on the participants' choices and hence was not controlled. Although colors were consistently associated with either small or large outcomes for each participant, there remained some degree of uncertainty about the outcome of each trial, because small outcomes varied between 6 and $11 \$$ and large outcomes varied between 32 and $40 \$$. Within these ranges, the frequency of possible outcomes was weighted such that the mean expected value of each trial was $+1 \notin$ for both small and large choices. The participants were not told about these experimental contingencies and were simply instructed to "use any strategy you want to help you maximize your points total." They were informed about their current cumulative winnings at the end of each block. There were 480 trials total, divided into 15 equal blocks. Thus, participants won $\$ 4.80$ on average. They were (truthfully) told that they would keep whatever money they won playing the game, in addition to their course credits.

EEG methods and analysis. We recorded the EEG using $\mathrm{Ag}-\mathrm{AgCl}$ electrodes embedded in a fabric cap (Neurosoft, El Paso, TX), arranged according to the 10-20 system, from channels FP1, FP2, AFz, F7, F3, Fz, F4, F8, FT7, FC3, FCz, FC4, FT8, T7, C3, Cz, C4, T8, TP7, CP3, CPz, $\mathrm{CP} 4, \mathrm{TP} 8, \mathrm{P} 7, \mathrm{P} 3, \mathrm{Pz}, \mathrm{P} 4, \mathrm{P} 8, \mathrm{O} 1, \mathrm{Oz}$, and $\mathrm{O} 2$. Other electrodes were placed on the right mastoid, above and below the right eye, and on the outer canthi of both eyes. The electrode common was placed on the chin. All electrode recordings were referenced to an electrode placed on the left mastoid, and electrode impedances were kept below $50 \mathrm{k} \Omega$. EEG data were amplified by a gain of 20,000 with a 12 bit processor using Sensorium (Charlotte, VT) EPA-6 128 Channel Electro-Physiology Amplifiers ( $1 \mathrm{G} \Omega$ input impedance). The sampling rate was $250 \mathrm{~Hz}$.

Separate EEG epochs of $800 \mathrm{msec}$ (200 msec baseline) were extracted off-line for chosen and alternative outcome stimuli on each trial. These EEG epochs were rereferenced to linked-mastoid electrodes and baseline corrected by subtracting half of the activity recorded at the right mastoid and the average activity of that channel during the baseline period separately for each EEG epoch recorded at each channel. Ocular artifacts were corrected with an eye-movement correction algorithm (Gratton et al., 1983).

We measured P300 amplitude on individual trials as the most positive peak in the waveform at electrode location $\mathrm{Pz}$ in the period 200-600 msec after feedback onset, after $2 \mathrm{~Hz}$ low-pass filtering. We quantified the feedback negativity in the averaged ERP waveforms for each participant as the base-to-peak difference in voltage between the most negative peak $200-400 \mathrm{msec}$ after stimulus onset and the average voltage of the immediately preceding and following positive peaks. We chose to include both the preceding and following positive peaks in our base-to-peak measure of the feedback negativity to avoid overestimating its amplitude in conditions in which this component peaked after the P300. Nevertheless, we found qualitatively similar results in an analysis in which we measured the feedback negativity as the voltage difference between the negative peak and the preceding positive peak alone, as used in many previous studies of the feedback negativity.

For the purposes of statistical analysis of scalp topographies, component amplitudes were calculated across 15 electrode locations that were chosen to cover scalp areas known from previous studies to be the focus of the feedback negativity and P300. Initially, the feedback negativity and P300 data were each subjected to ANOVAs with four within-subjects factors: reward magnitude (small and large), reward valence (win and loss), anteroposterior scalp location (frontal, frontocentral, central, centroparietal, parietal), and lateral scalp location $(3=$ left; $z=$ midline; $4=$ right). Subsequently, more detailed analyses focused on the electrode locations at which the components were maximal. In all analyses, degrees of freedom were corrected using Greenhouse-Geisser $\epsilon$ values.

\section{Results}

\section{Behavioral results}

There are few meaningful behavioral measures in the task, because rewards and penalties were assigned pseudorandomly. The 


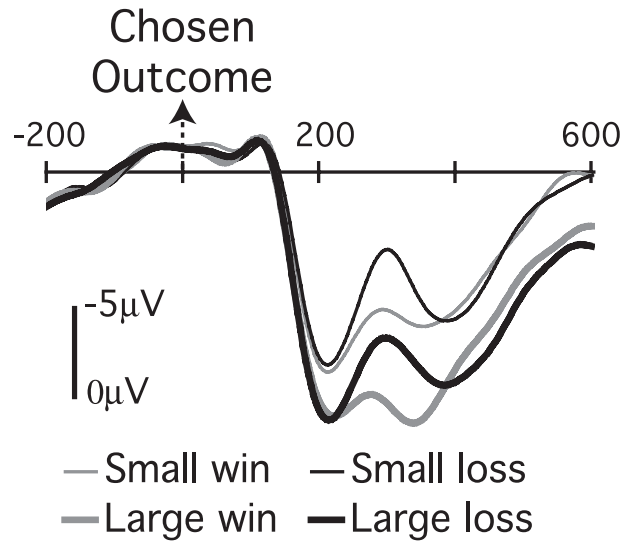

Figure 2. Grand-average ERP waveforms from channel FCz as a function of reward magnitude and valence of chosen outcomes. The abscissa shows the time (in milliseconds) relative to the presentation of the outcome stimulus.

task was simply intended as a realistic context in which the participants could experience rewards and penalties. Nevertheless, there was a good deal of variability across participants in their preferences for small or large decks. The proportion of large decks selected by participants varied between 0.38 and 0.66 (mean $=0.52 ; \mathrm{SD}=0.07)$. These differences reflected stable response strategies. We found a significant correlation between the proportion of small and large choices made by each participant in the first half and second half of the experiment $\left(r_{(15)}=\right.$ $0.5 ; p<0.05$ ). Moreover, the greater the proportion of small (or large) choices made by a participant, the faster they were to select a small (or large) card $\left(r_{(15)}=0.69 ; p<0.01\right)$.

Importantly, participants' choices indicated that they were sensitive to the outcomes of their gambles. Participants were more likely to select a large card if they had lost money on the previous trial than if they had won money $\left(F_{(1,15)}=7.1 ; p<0.05\right)$ (cf. Gehring and Willoughby, 2002). This was the case regardless of whether the loss on the previous trial was large or small $(F<1)$. Participants were also sensitive to the nature of the alternative outcome (what they would have won had they made a different choice). They were more likely to choose decks that on the previous trial had been associated with positive alternative outcomes $\left(F_{(1,15)}=11.9 ; p<0.01\right)$. In particular, participants were more likely to choose a large card if the alternative outcome on the previous trial was a large win than if this alternative was a large loss $\left(t_{(15)}=17.1 ; p<0.01\right)$. They were also slightly more likely to choose a small card when the alternative outcome on the previous trial was a small win than if this alternative was a small loss, but this effect was not reliable $(t<1)$.

\section{ERP results}

\section{Chosen outcomes}

Grand-average ERP waveforms for the four possible outcomes are shown in Figure 2. The data are shown for electrode location $\mathrm{FCz}$ at which the feedback negativity was maximal, as described below. Although P300 amplitude was maximal over more posterior locations, again as described below, a substantial P300 is nonetheless evident at FCz. Thus, consistent with previous research, ERP waveforms elicited by feedback stimuli were characterized by a large P300, on which was superimposed a feedback negativity peaking $\sim 300 \mathrm{msec}$ after feedback presentation. Figure

\section{Large minus small outcomes}
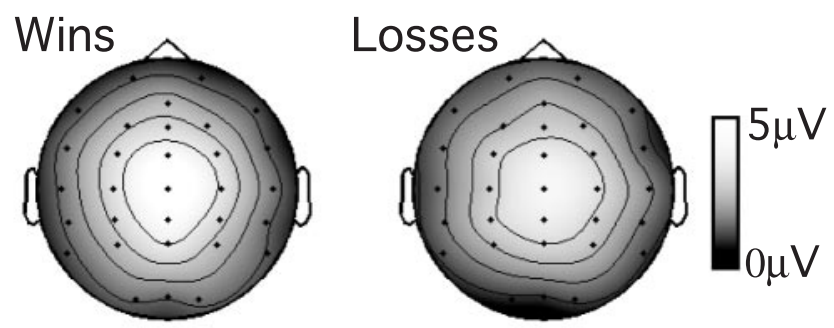

\section{Loss minus gain outcomes}
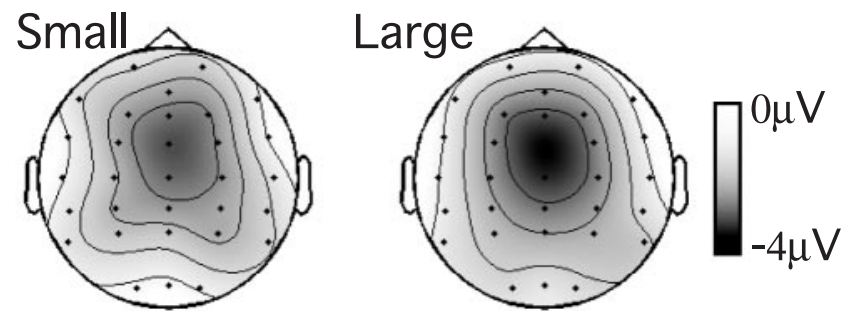

Figure 3. Scalp topography of orthogonal contrasts between reward magnitude and valence of chosen outcomes 300 msec after presentation of the outcome stimulus. Top, Topography of voltage differences between large and small outcomes separately for trials involving wins and losses. Bottom, Topography of voltage differences between losses and wins separately for trials with large and small outcomes.

3 shows scalp voltage maps of difference waves for orthogonal contrasts between reward valence and magnitude at this time.

P300. An ANOVA of P300 amplitude revealed reliable main effects of anteroposterior location $\left(F_{(4,60)}=15.7 ; \epsilon=0.34 ; p<\right.$ $0.01)$ and laterality $\left(F_{(2,30)}=9.3 ; \epsilon=0.82 ; p<0.01\right)$ and a marginally reliable interaction between these factors $\left(F_{(8,120)}=\right.$ 2.3; $\epsilon=0.61 ; p=0.05)$, indicating that P300 amplitude was greatest at posterior midline sites $(\mathrm{CPz}$ and $\mathrm{Pz})$ and reduced at more frontal and lateral electrode locations. There was a reliable main effect of reward magnitude $\left(F_{(1,15)}=28.6\right.$; $\left.p<0.01\right)$, indicating that P300 amplitude was greater for large outcomes than for small outcomes. As shown in Figure 3 (top), the effect of reward magnitude was greatest at the posterior midline sites at which the P300 was maximal, reflected in reliable interactions between reward magnitude and anteroposterior location $\left(F_{(4,60)}=16.1 ; \epsilon=0.37 ; p<0.01\right)$ and reward magnitude and laterality $\left(F_{(2,30)}=7.6 ; \epsilon=0.86 ; p<0.01\right)$. In contrast to these strong effects of reward magnitude, there was no reliable main effect or interactions involving the factor of reward valence on P300 amplitude ( $p$ values $>0.1)$.

Subsequent pairwise comparisons indicated that the effects of reward magnitude on P300 amplitude were reliably greater at posterior scalp locations $\mathrm{CPz}$ and $\mathrm{Pz}$ than at frontal sites $\mathrm{Fz}$ and FCz ( $p$ values $<0.01$ ). At electrode location Pz, P300 amplitude varied across conditions as follows: large loss, $18.4 \mu \mathrm{V}$; large win, $18.9 \mu \mathrm{V}$; small loss, $13.9 \mu \mathrm{V}$; small win, $13.7 \mu \mathrm{V}$. An ANOVA on the data from location $\mathrm{Pz}$ confirmed that P300 amplitude was reliably greater on trials with large outcomes than on trials with small outcomes $\left(F_{(1,15)}=29.5 ; p<0.01\right)$ but was unaffected by the valence of the outcome stimulus $(F<1)$ being equally large for losses and wins. The difference in P300 amplitude between large and small outcomes does not reflect a difference in the frequencies with which these outcomes were encountered. As 


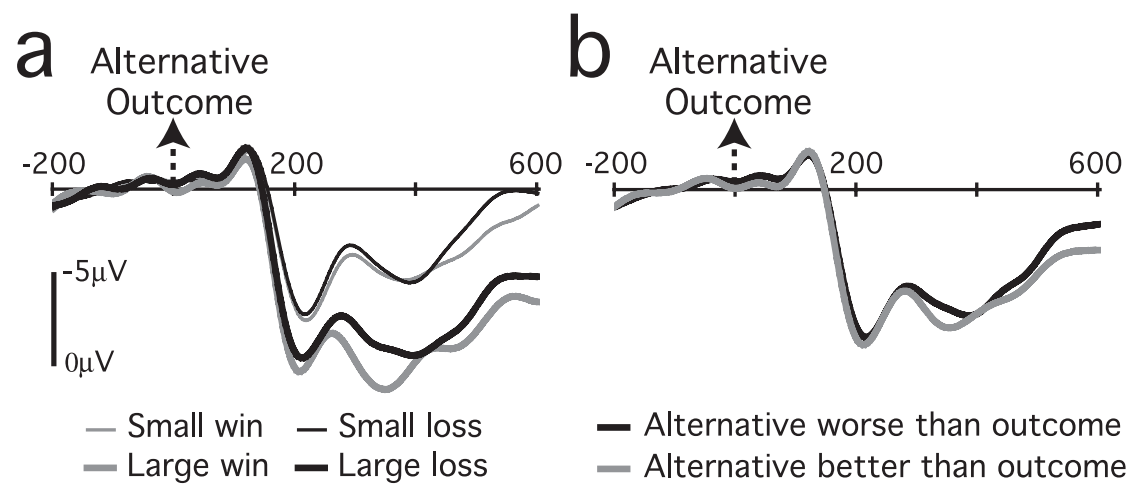

Figure 4. Grand-average ERP waveforms from channel FCz after the presentation of alternative outcomes. Results are shown separately as a function of the objective reward magnitude and valence of these outcomes $(a)$ and the value of these outcomes relative to the chosen outcome on the trial $(b)$. The abscissa shows the time (in milliseconds) relative to the presentation of the outcome stimulus.

\section{Large minus small alternatives}
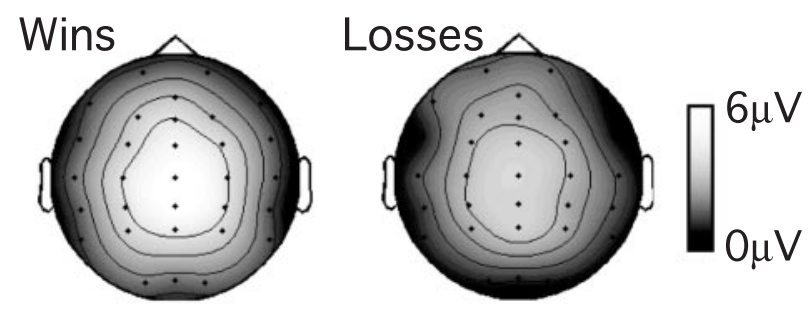

\section{Loss minus gain alternatives}
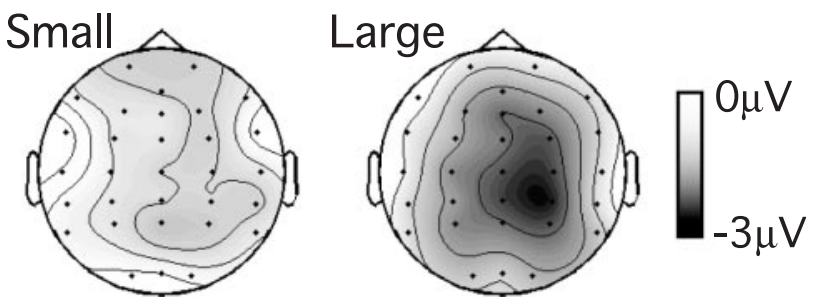

Figure 5. Scalp topography of orthogonal contrasts between reward magnitude and valence of alternative outcomes 300 msec after stimulus presentation. Top, Topography of voltage differences between large and small outcomes. Bottom, Topography of voltage differences between trials in which the alternative outcome was associated with gains and losses of money; trials with large and small outcomes are indicated separately.

noted above, small and large outcomes were encountered with approximately equal frequency. Moreover, when we performed a median split analysis to compare participants who chose mainly large decks with participants who chose mainly small decks, P300 amplitude differences between large and small outcomes were, if anything, slightly larger in the former group $(5.7 \mu \mathrm{V})$ than in the latter $(4.1 \mu \mathrm{V} ; t<1)$. P300 amplitude is typically high for unexpected or infrequent stimuli and low for expected or frequent stimuli (Sutton et al., 1967). Thus, the observed P300 amplitude difference between large and small outcomes cannot be explained in terms of the frequency with which outcomes were encountered and instead appears to relate specifically to the reward value of the feedback stimuli.

Feedback negativity. We quantified the feedback negativity as the base-to-peak difference in voltage between the most negative peak 200-400 msec after stimulus onset and the immediately preceding and following positive peaks. An ANOVA of these data revealed a reliable main effect of reward valence $\left(F_{(1,15)}=4.8 ; p<0.05\right)$, indicating that the feedback negativity was greater after losses than after gains. As shown at the bottom of Figure 3, this difference between loss and gain trials, the feedback negativity, was greatest at midline frontocentral locations, reflected in a reliable interaction between reward valence and anteroposterior location $\left(F_{(4,60)}=8.1 ; \epsilon=0.42 ; p<\right.$ $0.01)$ and marginally reliable interactions between valence and laterality $\left(F_{(2,30)}=\right.$ 3.0; $\epsilon=0.98$; $p=0.06)$ and between valence, anteroposterior location, and laterality $\left(F_{(8,120)}=2.3 ; \epsilon=0.54 ; p=0.06\right)$. In contrast to these effects of reward valence, there was no significant effect of reward magnitude on the amplitude of the feedback negativity $(F<1)$.

Pairwise comparisons indicated that the effects of reward valence on the amplitude of the feedback negativity were reliably greater at frontal scalp locations Fz and FCz than at posterior sites $\mathrm{Pz}$ and $\mathrm{CPz}$ ( $p$ values $<0.01)$. Subsequent analyses focused on electrode location $\mathrm{FCz}$, at which the feedback negativity was maximal. At $\mathrm{FCz}$, the amplitude of this component was larger after losses $(-6.4 \mu \mathrm{V})$ than after gains $\left(-3.5 \mu \mathrm{V} ; F_{(1,15)}=14.0 ; p<\right.$ 0.01 ). Critically, the feedback negativity was no larger after a large loss $(-6.0 \mu \mathrm{V})$ than after a small loss $(-6.7 \mu \mathrm{V} ; F<1)$. To confirm that this finding was not an artifact of the base-to-peak algorithm used, we also quantified the feedback negativity as the most negative peak in the difference wave between losses and gains and as the average voltage in a $48 \mathrm{msec}$ window centered around the component peak. Neither analysis revealed a reliable difference between the feedback negativity elicited by large and small losses $(F<1.25 ; p$ values $>0.25)$.

Summary. The P300 and feedback negativity were clearly evident in the ERP waveforms. The P300 had a posterior midline focus, whereas the feedback negativity was maximal over medial frontal sites. Critically, we found a functional dissociation between these two reward-sensitive components, with the P300 selectively sensitive to reward magnitude, regardless of reward valence, and the feedback negativity showing the opposite pattern. Alternative outcomes

Additional functional dissociations between the P300 and feedback negativity are suggested by an analysis of the ERP waveforms elicited by stimuli indicating the value of the unchosen card. Figure 4 presents grand-average ERP waveforms at $\mathrm{FCz}$ after alternative outcomes as a function of the absolute reward value associated with those stimuli (Fig. $4 a$ ) and as a function of their value relative to the chosen outcome (Fig. $4 b$ ). Figure 5 shows scalp voltage maps of difference waves for orthogonal contrasts between reward valence and magnitude at $300 \mathrm{msec}$ after presentation of the alternative outcome.

P300. As was the case for chosen outcomes, P300 amplitude was greatest at midline posterior scalp locations, reflected in reliable main effects of anteroposterior location $\left(F_{(4,60)}=16.4 ; \epsilon=\right.$ $0.29 ; p<0.01)$ and laterality $\left(F_{(2,30)}=9.1 ; \epsilon=0.97 ; p<0.01\right)$ and a reliable interaction between these two factors $\left(F_{(4,60)}=3.1 ; \epsilon=\right.$ $0.51 ; p<0.05)$. Critically, P300 amplitude was greater when the alternative outcome was large than when the alternative was small $\left(F_{(1,15)}=27.0 ; p<0.01\right)$. This difference between large and small alternative outcomes showed a centroparietal focus (Fig. 5, top), indicated by reliable interactions between reward magni- 
tude and anteroposterior location $\left(F_{(4,60)}=4.8 ; \epsilon=0.28 ; p<\right.$ $0.05)$ and reward magnitude and laterality $\left(F_{(2,30)}=18.8 ; \epsilon=\right.$ $0.83 ; p<0.01)$. At electrode location $\mathrm{Pz}, \mathrm{P} 300$ amplitude was much greater after large $(20.2 \mu \mathrm{V})$ than small $(14.2 \mu \mathrm{V})$ alternative outcomes $\left(F_{(1,15)}=22.3 ; p<0.01\right)$ (comparable effects on P300 amplitude can be seen in Fig. $4 a$ for electrode FCz). Together, these findings suggest that P300 amplitude is sensitive to the magnitude of signaled reward value, regardless of whether the reward was actually obtained.

Surprisingly, P300 amplitude also showed some sensitivity to the valence of alternative outcomes, being reliably larger when the alternative outcome was a gain than when it was a loss $\left(F_{(1,15)}=5.3 ; p<0.05\right)$. This effect of valence was particularly marked for large outcomes, reflected in a reliable three-way interaction between reward valence, magnitude, and anteroposterior location $\left(F_{(4,60)}=5.7 ; \epsilon=0.43 ; p<0.05\right)$. A separate analysis indicated that P300 amplitude was also larger when the alternative outcome was better than the chosen outcome than when the alternative outcome was the worse of the two $\left(F_{(1,15)}=5.0 ; p<\right.$ 0.05). As shown at the bottom right of Figure 5, the effects of reward valence were largest over posterior scalp locations (although corresponding effects can also be observed in Fig. 4 for electrode location FCz). Figure 5 also suggests that the effects of reward valence on P300 amplitude showed some evidence of right lateralization. However, there were no reliable interactions involving reward valence and laterality ( $p$ values $>0.25$ ). Thus, it remains an open question whether these effects of reward valence reflect processing in brain regions separate from those responsible for coding reward magnitude, as reflected in the midline centroparietal P300 effects described above.

Feedback negativity. The feedback negativity was insensitive to the reward value of alternative outcomes. When quantified across all 15 scalp locations using the base-to-peak measure described above, the amplitude of the feedback negativity showed no main effects of reward valence or magnitude $(F$ values $<1)$ and no reliable interaction between these two factors $\left(F_{(1,15)}=1.3 ; p>\right.$ 0.25). Likewise, at electrode $\mathrm{FCz}$, at which the feedback negativity to chosen outcomes was maximal, there was no effect of whether the alternative outcome was a loss $(-4.4 \mu \mathrm{V})$ or gain $(-4.3 \mu \mathrm{V})$ of money $(F<1)$ and no effect of reward magnitude $(F<1)$ (Fig. $4 a)$. Nor did the feedback negativity show evidence of sensitivity to the value of alternative outcomes in relation to chosen outcomes (Fig. $4 b$ ). The feedback negativity was as large when the alternative outcome was worse than the chosen outcome $(-4.0$ $\mu \mathrm{V})$ as when the alternative outcome was better than the chosen outcome $(-3.9 \mu \mathrm{V} ; F<1)$. Thus, the feedback negativity was insensitive to whether the participants had made the correct or incorrect choice on the trial.

Summary. The P300, but not the feedback negativity, showed sensitivity to information about the reward value of alternative outcomes. As was the case for chosen outcomes, P300 amplitude was greater for large than small alternative outcomes. P300 amplitude also showed an unexpected sensitivity to the valence of alternative outcomes, being greatest on trials in which participants had failed to select a card associated with a large win. In contrast, the feedback negativity was unaffected by whether the alternative outcome was a win or loss, large or small, or better or worse than the chosen outcome.

\section{ERPs and behavioral adjustments}

The findings described above demonstrate that the feedback negativity is observed when participants' choices result in a loss of money and that participants are more likely to choose large (or risky) gambles after such losses. The results also demonstrate an increased positivity over right posterior regions when participants' choices led them to miss out on a large win (the alternative outcome on the trial). After such trials, participants were more likely to select large decks. Thus, chosen and alternative outcomes were associated with specific ERP signatures and particular behavioral adjustments.

To assess whether these behavioral and ERP phenomena are related, we performed two additional analyses. In the first analysis, we divided the participants into two groups on the basis of the size of their behavioral adjustment after losses of money on the chosen outcome. We then compared the amplitude of the feedback negativity elicited by such losses across the two groups. This analysis revealed that participants whose behavior showed a greater shift to risk-taking behavior after losses of money had a greater feedback negativity $(-8.9 \mu \mathrm{V})$ than participants who showed little behavioral adjustment after losses $\left(-3.9 \mu \mathrm{V} ; t_{(14)}=\right.$ $2.7 ; p<0.05)$. In the second analysis, we divided the participants into two groups on the basis of the size of their behavioral adjustment after trials in which they failed to select a card associated with a large win. We then compared these two groups according to the difference in P300 amplitude elicited by large-gain and large-loss alternative outcomes. This P300 effect was larger in the participants showing a greater behavioral adjustment $(3.9 \mu \mathrm{V})$ than in participants showing small behavioral adjustments $(0.6$ $\mu \mathrm{V})$, although the difference was only marginally reliable $\left(t_{(14)}=\right.$ 1.8; $p=0.09)$. Together, these results suggest that individual differences in the feedback negativity and P300 reflect meaningful differences in the processing of reward information.

\section{Discussion}

Consistent with previous research, we found sensitivity to information about rewards and penalties in two ERP components, the P300 and feedback negativity. Extending these findings, we found a double dissociation between the two components in relation to chosen outcomes, with the P300 sensitive to reward magnitude but unaffected by reward valence and the feedback negativity showing the opposite pattern. These findings suggest that reward magnitude and valence are processed separately in the brain. Moreover, this separation appears to occur very early in processing; effects of reward magnitude and valence were apparent on the P300 and feedback negativity within 300 msec of feedback onset.

Analyses of ERP waveforms elicited by unchosen, alternative outcomes suggested additional functional dissociations between the two components. Although these alternative outcomes were not directly associated with rewards and penalties, they were nevertheless behaviorally relevant. This behavioral relevance was reflected in the finding that participants adjusted their choices based on alternative outcomes, being more likely to select large (risky) decks if on the previous trial they had failed to select a card associated with a large reward. It is therefore of interest that the P300, but not the feedback negativity, was affected by the value of alternative outcomes. P300 amplitude increased with the magnitude of alternative outcomes, whereas the feedback negativity was insensitive both to the valence of alternative outcomes and to whether these alternative outcomes were better or worse than chosen outcomes.

The observed influence of reward magnitude on P300 amplitude is not attributable to differences in the relative frequencies of large and small outcomes. If anything, participants chose large outcomes more often than small ones. Nor does the effect appear to reflect a general increase in EEG amplitude because more at- 
tention is paid to gambles with large outcomes. If this were so, one would expect the amplitude of the feedback negativity to also increase with the magnitude of the penalty incurred, but this was not observed. Indeed, we observed a null effect of reward magnitude on the feedback negativity, although voltage differences in P300 amplitude as a function of magnitude appeared before its onset. Thus, the impact of reward magnitude on the P300 appears to reflect a meaningful change in neural processing. In this regard, the finding that P300 amplitude was affected by the reward magnitude of both chosen and alternative outcomes might be interpreted as evidence that this component reflects an objective coding of reward magnitude, regardless of whether the reward is actually obtained. However, given that alternative outcomes were behaviorally relevant to the task, it could also be that P300 amplitude varies with reward magnitude because of the increased motivational or affective significance of greater rewards and penalties. Thus, the mechanism by which the P300 is sensitive to reward magnitude remains an issue for future research.

It has been reported previously that a larger P300 is observed in response to affectively negative images than in response to positive images that are matched according to subjective ratings of arousal (Ito et al., 1998), suggesting that the P300 is sensitive to the valence of stimuli. This result initially seems at odds with the present finding that the valence of chosen outcomes does not affect the P300 and seems easier to reconcile with our finding of an increased positivity over right posterior scalp regions when alternative outcomes were better than chosen outcomes (i.e., after feedback indicating that participants had made the incorrect choice). One possible characterization of these findings is that effects of valence on the P300 are observed when valence is defined in terms of high-level affective evaluations, for example, reflecting regret or disappointment (at having made an incorrect choice) or disgust (in response to affectively negative images), but not when valence is defined in terms of straightforward reward value.

Thus, an important implication of our findings is that multiple evaluative processes may contribute to P300 generation. An issue for future research will be to tease apart these processes and to distinguish the neural mechanisms underlying them. Neuroimaging studies have already identified a number of regions that are sensitive to reward magnitude, including the orbitofrontal cortex, insula, and ventral striatum (Elliott et al., 2000; Knutson et al., 2000; Breiter et al., 2001; Delgado et al., 2003). It is possible that a number of these cortical sources contribute to the broadly distributed effect of reward magnitude observed here, an issue that might be addressed using high-density recording methods and EEG decomposition analyses. These approaches should also provide insight into the neural basis of the effects we observed with alternative outcomes, which elicited an increased positivity when they indicated incorrect response choices. Our data provide tentative evidence of right lateralization of these effects, a finding that might be pursued in future research.

The most likely neural generator of the feedback negativity, given its frontocentral focus, is the anterior cingulate cortex (Miltner et al., 1997; Gehring and Willoughby, 2002; Holroyd and Coles, 2002), an area that is consistently activated in neuroimaging studies of reward processing (Elliott et al., 2000; Knutson et al., 2000; Delgado et al., 2003). Consistent with the present findings, this area has been shown to be more sensitive to negative than positive outcomes (Knutson et al., 2000; Delgado et al., 2003), and recent evidence suggests that it is relatively insensitive to reward magnitude (Delgado et al., 2003). Thus, converging evidence suggests that the anterior cingulate cortex forms part of a system responsible for evaluating the reward value and motivational significance of ongoing events (Gehring and Willoughby, 2002; Holroyd and Coles, 2002; Nieuwenhuis et al., 2004). However, the present findings indicate that evaluative information processed by the anterior cingulate cortex is somewhat limited, being insensitive to the magnitude of experienced rewards, to the value of rewards that were not directly experienced, and to the relative value of chosen and alternative outcomes.

These findings suggest important constraints on theories of anterior cingulate function and the feedback negativity. Most obviously, theories that associate the feedback negativity with "instant utility" (Gehring and Willoughby, 2002) or changes in expected value (Holroyd and Coles, 2002) would appear to predict that a greater negativity should be observed after larger monetary losses. The present findings of a null effect of reward magnitude on the feedback negativity contradict these predictions. To explain our findings, one might propose that the evaluative process is simply rather coarse in nature, providing a discrete evaluation of events as good or bad regardless of magnitude. Alternatively, the evaluative process might scale reward by the expected magnitude of the outcomes (Holroyd et al., 2004). In the present study, reward magnitude could be predicted to some degree, because particular card colors were consistently associated with large or small outcomes. Either way, the present findings make the important point that the feedback negativity does not reflect a simple scalar evaluation of reward value.

The present findings of behavioral adjustments and their relationship to neural activity also have implications for current theories. We found that participants were more likely to choose large, risky options immediately after experiencing losses, and that a greater feedback negativity to losses was observed in participants who showed a greater shift to risk-taking behavior after such losses. That is, after a large loss of money, participants tended to repeat the response choice that led to that outcome (selection of the large, risky option), particularly if the outcome elicited a large feedback negativity. This finding presents a challenge to the hypothesis that the feedback negativity reflects a process of associative reinforcement learning (Holroyd and Coles, 2002), which predicts that participants should be less likely rather than more likely to repeat response choices that led to negative outcomes and a feedback negativity. Instead, the result provides evidence of higher-level strategic adjustments. It seems that participants attempted to compensate for experienced losses by taking short-term risks in the hope of large wins, thus falling prey to the gambler's fallacy. Our findings suggest that the feedback negativity provides an effective index of the neural processing underlying these strategic adjustments in behavior.

Interestingly, evidence of associative learning effects were apparent in participants' responses to alternative outcomes. Participants were more likely to gamble on large, risky outcomes if on the previous trial they had missed out on a large win. That is, they were more likely to select response options that were recently associated with positive (alternative) outcomes. However, such events were not accompanied by a feedback negativity, but were instead associated with an increased positivity over posterior scalp regions, perhaps somewhat lateralized to the right hemisphere. An important goal for future research will be to extend the present results, in which the amplitude of this scalp positivity was only marginally reliably associated with behavioral adjustments, and to develop theories of the relationship between the various evaluative processes reflected in the ERP. 


\section{References}

Breiter HC, Aharon I, Kahneman D, Dale A, Shizgal P (2001) Functional imaging of neural responses to expectancy and experience of monetary gains and losses. Neuron 30:619-639.

Delgado MR, Locke HM, Stenger VA, Fiez JA (2003) Dorsal striatum responses to reward and punishment: effects of valence and magnitude manipulations. Cogn Affect Behav Neurosci 3:27-38.

Elliott R, Friston KJ, Dolan RJ (2000) Dissociable neural responses in human reward systems. J Neurosci 20:6159-6165.

Gehring WJ, Willoughby AR (2002) The medial frontal cortex and the rapid processing of monetary gains and losses. Science 295:2279-2282.

Gratton G, Coles MGH, Donchin E (1983) A new method for off-line removal of ocular artifact. Electroencephalogr Clin Neurophysiol 55:468-484.

Holroyd CB, Coles MGH (2002) The neural basis of human error processing: reinforcement learning, dopamine, and the error-related negativity. Psychol Rev 109:679-709.

Holroyd CB, Larsen JT, Cohen JD (2004) Context dependence of the eventrelated brain potential associated with reward and punishment. Psychophysiology 41:245-253.
Ito T, Larsen JT, Smith NK, Cacioppo JT (1998) Negative information weighs more heavily on the brain: the negativity bias in evaluative categorizations. J Pers Soc Psychol 75:887-900.

Johnston VS (1979) Stimuli with biological significance. In: Evoked brain potentials and behavior (Begleiter H, ed), pp 1-12. New York: Plenum.

Knutson B, Westdorp A, Kaiser E, Hommer D (2000) fMRI visualization of brain activity during a monetary incentive delay task. NeuroImage $12: 20-27$.

Miltner WHR, Braun CH, Coles MGH (1997) Event-related potentials following incorrect feedback in a time-estimation task: evidence for a "generic” neural system for error detection. J Cogn Neurosci 9:788-798.

Nieuwenhuis S, Yeung N, Holroyd CB, Schurger A, Cohen JD (2004) Sensitivity of electrophysiological activity from medial frontal cortex to utilitarian and performance feedback. Cereb Cortex 14:741-747.

Sutton S, Tueting P, Zubin J, John ER (1967) Information delivery and the sensory evoked potential. Science 155:1436-1439.

Sutton S, Tueting P, Hammer M, Hakerem G (1978) Evoked potentials and feedback. In: Multidisciplinary perspectives in event-related potential research (Otto D, ed), pp 184-188. Washington, DC: United States Government Printing Office. 